\title{
Quantitative determination of a nano-object's atom density without atomic resolution
}

\author{
Christopher Zaum, ${ }^{1}$ Jörg Meyer, ${ }^{2, *}$ Karsten Reuter, ${ }^{2}$ and Karina Morgenstern ${ }^{3, \dagger}$ \\ ${ }^{1}$ Leibniz Universität Hannover, Institut für Festkörperphysik, Appelstr. 2, D-30167 Hannover, Germany \\ ${ }^{2}$ Department Chemie, Technische Universität München, Lichtenbergstraße 4, D-85748 Garching, Germany \\ ${ }^{3}$ Ruhr-Universität Bochum, Lehrstuhl für physikalische Chemie I, Universitätsstr. 150, D-44801 Bochum, Germany
}

(Received 20 September 2014; published 15 October 2014)

\begin{abstract}
We outline a possibility to determine the adatom density of individual nano-objects via measurement of their electronic structure. For this aim, the nonlinear shift of image potential states measured on individual $\mathrm{Cu}$ nanoclusters on $\mathrm{Ag}(100)$ by low-temperature scanning tunneling spectroscopy is carefully analyzed. The quantitative analysis is confirmed by density-functional theory calculations. A peak width analysis furthermore reveals whether the clusters are purely metallic or alloyed.
\end{abstract}

DOI: 10.1103/PhysRevB.90.165418

For nanotechnology a structural control on the nanoscale is essential, because the physical as well as chemical properties of nanoscale objects depend on the exact arrangement of their atoms [1]. As a first step, it is of vital importance for scientists and engineers to resolve the internal structure of the nanosystems to understand and, in a subsequent step, tune the resulting macroscopic properties. The atomic structure of nanoparticles can be determined directly by scanning transmission microscopy (STEM) with high resolution down to $50 \mathrm{pm}$ [2], which shows a two-dimensional projection of the crystal in some high-index direction of particles. With STEM, nanoparticles were imaged three dimensionally [3]. Even single defects within nanoparticles were identified recently [4]. Due to the width of the electron beam, the extraction of the data demands sophisticated procedures.

For two-dimensional nanoparticles on surfaces, scanning tunneling microscopy (STM) turned out to be a simple and versatile tool for high resolution imaging. However, atomic resolution is often impossible for discrete entities consisting of only a few atoms, in particular for nanoclusters. These objects have the tendency to be moved or restructured at the tunneling parameters necessary for atomic resolution. On the other hand, structural information of a metal surface is clearly reflected in the energetic positions of its image potential states [5,6]. A Rydberg-like series of these states emerges, when an outside charge polarizes a metal surface and is attracted to the induced polarization charge. As the energy levels of these states are pinned to the vacuum level, they are closely related to the work function, which in turn varies with both the chemical composition of the surface and the surface orientation, and thus with atom density. Surface averaged values of energy, dispersion (and lifetime) of image potential states were extensively probed, first by inverse photoemission (IPES) [7] and later by two-photon photoemission spectroscopy (2PPE) [8]. High spatial resolution of an image potential state was achieved by scanning tunneling spectroscopy (STS) $[9,10]$ based on the pioneering works of Becker et al. [11] and Binnig et al. [12]. Such measurements provided information about

\footnotetext{
${ }^{*}$ Present address: Leiden Institute of Chemistry, Gorlaeus Laboratories, Leiden University, P.O. Box 9502, NL-2300 RA Leiden, The Netherlands.

${ }^{\dagger}$ karina.morgenstern@ rub.de
}

PACS number(s): 68.35.bd, 68.37.Ef, 68.43.Fg, 73.21.-b local variations of the electrostatic potential. Similar information is acquired by measuring contact potential differences by Kelvin probe force microscopy. For this method submolecular resolution was presented only recently [13], but the method has not yet been applied to nanoclusters. On the other hand, STS investigations of such structures yielded significant shifts of the energetic position of image potential states over the nanoislands [14], as well as a quantization due to stacking faults [15]. The shifts are consistent with a lateral confinement of the electrons as described by textbook (two-dimensional) particle-in-a-box models [14]. Such an assignment assumes atomically flat islands with perfect coordination, though a relation to potential relaxation or reconstruction of the latter has not been made so far [16].

In this article, we present a method for the analysis of metallic nanostructures on the nanometer scale based on the local measurement of image potential states. After the subtraction of confinement-induced effects, we utilize the fact that the image potential state depends on the local adatom density to resolve this density for islands consisting of only about ten atoms. As a model system we investigate nanoclusters created by the deposition of $\mathrm{Cu}$ on $\mathrm{Ag}(100)$ at room temperature, for which atomic resolution is hitherto only possible for islands pinned to surface defects [17]. We focus on islands smaller than $5 \mathrm{~nm}^{2}$ that are pure copper and known to be reconstructed due to strain effects [17].

The measurements are performed with a custom built low-temperature scanning tunneling microscope (STM) [18] in a UHV environment (base pressure $\leqslant 2 \times 10^{-10}$ mbar). The $\operatorname{Ag}(100)$ surface is cleaned by standard sputtering and annealing cycles. After cleaning, 0.01 to 0.05 monolayers (ML) of copper are deposited with deposition rates between 0.01 and $0.03 \mathrm{ML} / \mathrm{min}$ onto the substrate at room temperature (RT). The substrate is kept at RT for an additional $10 \mathrm{~min}$ to enable further island ripening [19]. After this time, the substrate is rapidly quenched to the measurement temperature of $5 \mathrm{~K}$. This procedure leads to an alloyed surface covered by nanosized clusters of quadratic shape [17]. The clusters consist of a single adatom layer and their areas range between 1 and $35 \mathrm{~nm}^{2}$. They are called islands in the following. Their size is determined at $50 \%$ value of the step height corresponding to the effective boundary of likewise metallic $\mathrm{Ag}(111)$ islands [20]. In contrast to other systems studied in our laboratory [21,22], attempts to achieve atomic resolution with 
STM tips modified by atomic or molecular adsorbates [23,24] have not been successful for the system studied here.

The local density of states of the metallic structures is probed by two types of STS measurements: $d z / d V$ spectroscopy with a closed feedback loop for large energy ranges and differential conductivity $(d I / d V)$ spectroscopy with an open feedback loop for high energy resolution [25]. The $d I / d V$ spectroscopy is utilized in quantitative measurements to avoid peak shifts [26]. Spatially resolved $d z / d V$ spectroscopy consists of a series of STM images acquired at increasing bias voltage $(V=50 \mathrm{mV})$ at the same position of the surface. After correcting for a small residual lateral drift, each pixel of the image series contains a $z(V)$ curve. These $z(V)$ curves are numerically differentiated to obtain the $d z / d V$ value for each point and for each bias voltage of the image series.

The differential conductivity spectroscopy is based on $I(V)$ curves recorded with an open feedback loop. Increasing the bias voltage in this operation mode is only possible by increasing the tip-sample distance simultaneously to prevent an overloading of the tunneling current amplifier. Thus, the measured current $I(V, \Delta z)$ depends not only on the bias voltage $V$, but also on the tip retraction $\Delta z \propto V$. To correct the influence of $\Delta z$, a correction function is deduced from calibration measurements performed on pure $\mathrm{Ag}(100)$ and $\mathrm{Cu}(111)$ surfaces and applied prior to the numerical differentiation. Due to the exponential increase of the differential conductivity with increasing bias voltage, the normalized differential conductivity $(d I / d V) /(I / V)$ is used for analysis [27]. The fact that no $\Delta z$ correction function is applied to the $d z / d V$ measurements leads to different absolute peak positions in the two different spectroscopy methods.

The determination of the image potential state properties by STS is hampered by the fact that the electric field applied between the sample and the tip deforms the potential at the solid-vacuum interface, leading to both an energy upshift [28,29] and a line broadening [30] of the image potential state. The amount of energy upshift (Stark shift) thereby differs for image potential states of different order [29], such that the natural $1 / n^{2}$ energy spacing is not observed in STS [1]. Stark-shifted image potential states (S-IPS) are often named field emission resonances and are published for the two surfaces of relevance here, $\operatorname{Ag}(100)$ [31] and $\mathrm{Cu}(111)$ [32]. Fortunately, their relative changes in energy and line width are much less affected than absolute values. In particular, relative changes within the 2nd Stark-shifted state are the least affected by the field [10].

Figure 1(a) shows a STM image of two differently sized islands, for which spatially resolved $d z / d V$ spectroscopy is performed. A two-dimensional (2D) cross section at $V=$ $6.1 \mathrm{~V}$ of the $d z / d V(x, y, V)$ scalar field is shown in Fig. 1(b). At this voltage the spatial distribution of the $d z / d V$ signal clearly differs for the two islands. While a high intensity region is located at the position of the small island, only the borders of the large island contribute to the signal [33]. In order to understand these differences, it is necessary to analyze not only the spatial dependence of the $d z / d V$ signal but also the voltage dependence.

Such voltage dependence across the large and the small islands is shown in Fig. 2. In both $d z / d V(x, y, V)$ cross
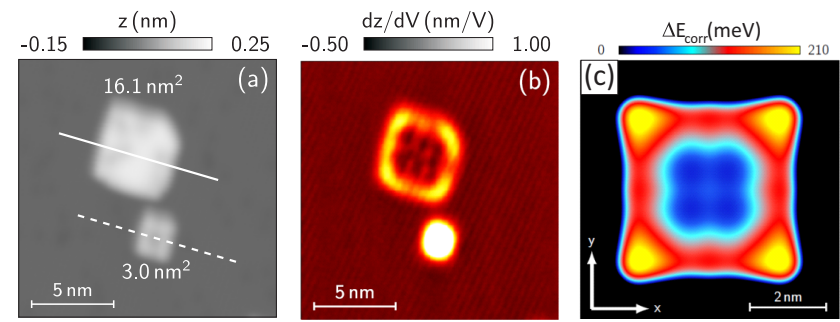

FIG. 1. (Color online) Topography, electronic structure, and confinement effect: (a) Topography image of a small $\left(3.0 \mathrm{~nm}^{2}\right)$ and a large island $\left(16.1 \mathrm{~nm}^{2}\right)(I=44 \mathrm{pA}, V=0.2 \mathrm{~V})$. (b) Spatially resolved $d z / d V$ intensity at a $6.1 \mathrm{~V}$ bias voltage $(I=44 \mathrm{pA}, V=6.1 \mathrm{~V})$. (c) $2 \mathrm{D}$ representation of the confinement effect correction function for an island of $20 \mathrm{~nm}^{2}$.

sections shown, three states are clearly visible at $V_{1} \approx 4.5$, $V_{2} \approx 5.8$, and $V_{3} \approx 6.7 \mathrm{~V}$. At the center of the large island, the states are shifted slightly towards higher energies $(\Delta V \approx$ $0.05 \mathrm{~V}$ ). This shift with $\Delta V \approx 0.3 \mathrm{~V}$ is noticeably larger for the small island.

Consequently, Fig. 2 elucidates the spatial distribution of the $d z / d V$ signal shown in Fig. 1(b): The bias voltage $V=$ $6.1 \mathrm{~V}$ corresponds to the energetic position of the 2nd S-IPS on the small island, yielding the high $d z / d V$ intensity at the position of the latter. In contrast, as there is no S-IPS located at $6.1 \mathrm{~V}$ on the large island, a low $d z / d V$ intensity is observed. The island's electronic structure thus depends on island size.

Note that the data presented in Fig. 2 is not corrected for the changes in the electric field due to the tip displacement. Because of the nonlinear $z$ retraction at the activated feedbackloop, the corresponding changes in shift are not correctable with reasonable effort. In contrast, for the linear $z$ retraction during $d I / d V$ measurements, a normalization is feasible and thus is a quantitative analysis of the data. Note that the
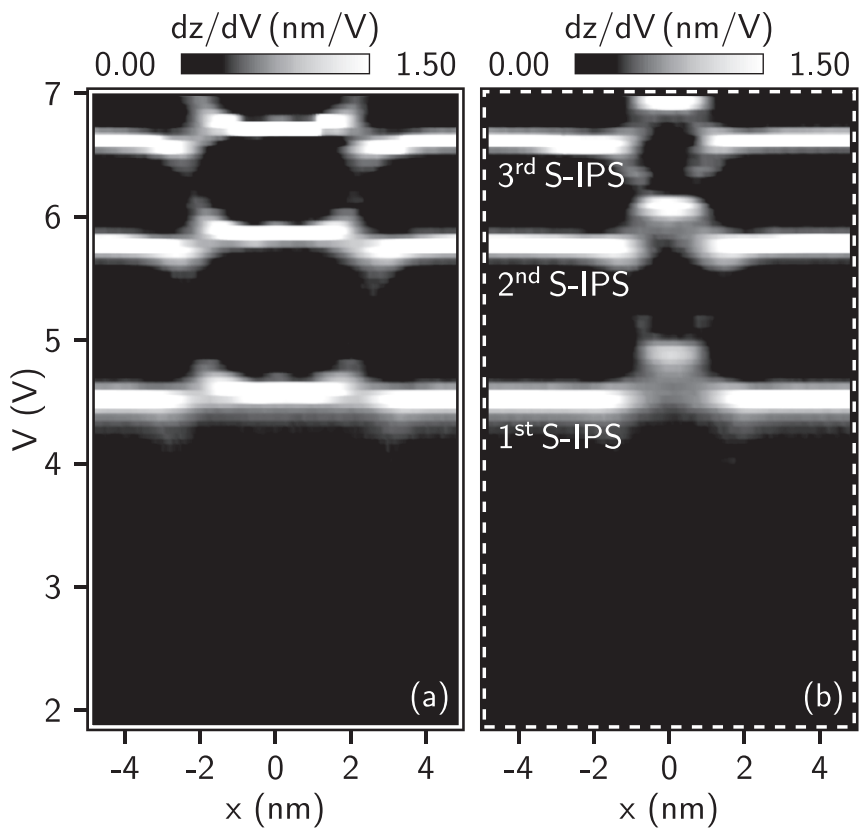

FIG. 2. Voltage dependence of $d z / d V$ along a line across the (a) large and (b) small islands in Fig. 1(a) as indicated there by the solid and dashed lines, respectively. 


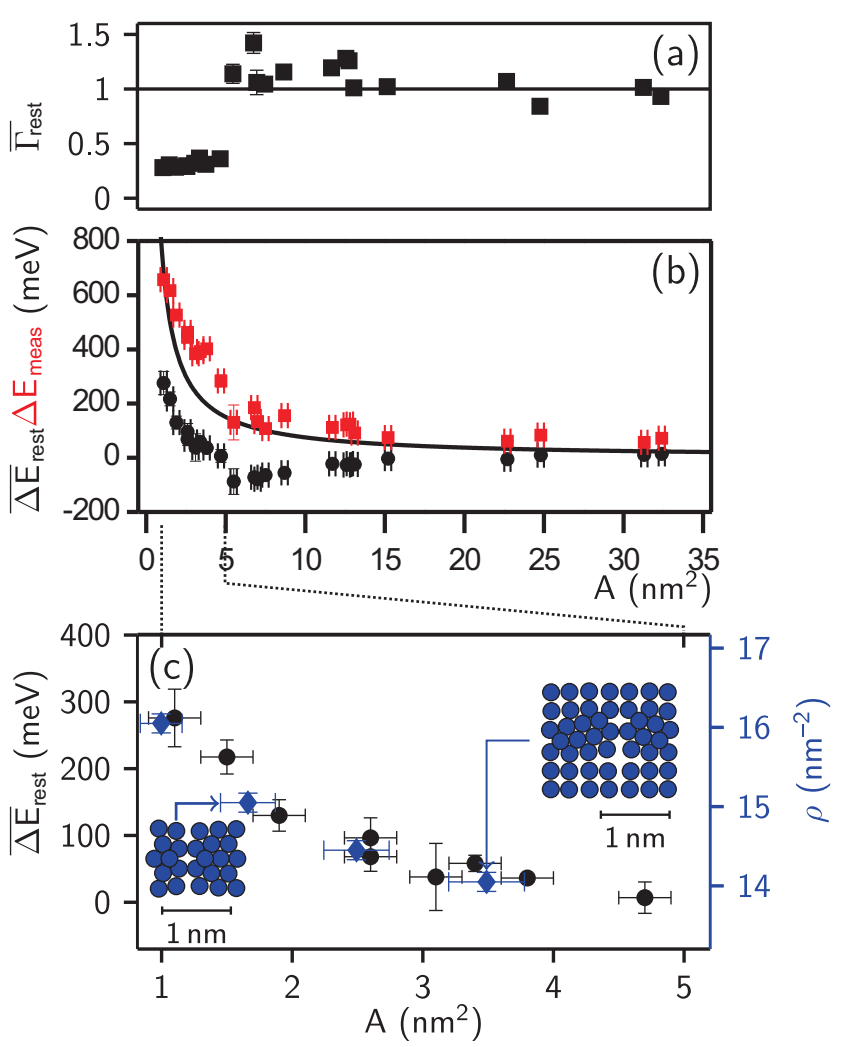

FIG. 3. (Color online) Dependence of 2nd S-IPS on the island size $A$ with respect to the terrace value. (a) Relative line width $\bar{\Gamma}_{\text {rest }}$. (b) Energy shift as measured ( $\Delta E_{\text {meas }}$, red squares) and without the confinement effect $\left(\overline{\Delta E}_{\text {rest }}\right.$, black circles). Black line is the expected energy shift for the quantum size confinement deduced from a simple particle-in-a-box model for square-shaped boxes $\Delta E_{\mathrm{box}}=\frac{\pi^{2} \hbar^{2}}{2 m^{*}} \frac{n_{x}^{2}+n_{y}^{2}}{A}$ with an effective mass parameter $m^{*}=m_{e}$ with $m_{e}$ the electron mass and $n_{x}=n_{y}=1$. (c) Zoom into the remaining energy shift $\left(\overline{\Delta E}_{\text {rest }}\right)$ for the smallest islands after the confinement subtraction (black circles, left axis) and island atom density as deduced from density-functional theory (DFT) calculations [17,27] (gray (blue) diamonds, right axis). Error bar on the $y$ axis reflects the energy spread across individual islands. Insets: Island structure as calculated by the DFT for $\mathrm{Cu}$ islands with 25 and 49 atoms on the left and on the right, respectively.

normalized data remains Stark shifted, and thus the $1 / n^{2}$ energy spacing of image potential states without perturbation by an electric field cannot be expected.

Figure 4(b) shows exemplarily three normalized $d I / d V$ spectra recorded along a line across two islands of different sizes. These spectra contain the aspired information about the atom density. To extract this information, first, the states of all spectra are fitted by Lorentzian functions. We here concentrate on the 2nd image potential state, because it is least affected by the applied external field as mentioned above [10]. Then, the data has to be corrected for the quantum size confinement effects induced by the presence of step edges as described in detail below. This procedure leads to a nearly constant and thus well-defined value on the terrace and on both islands $E_{\text {rest }}$ [Fig. 4(c)]. The same holds for the line width (not shown).

The measured average energy shift [Fig. 3(b)] shows an island size dependence that at first glance resembles the one

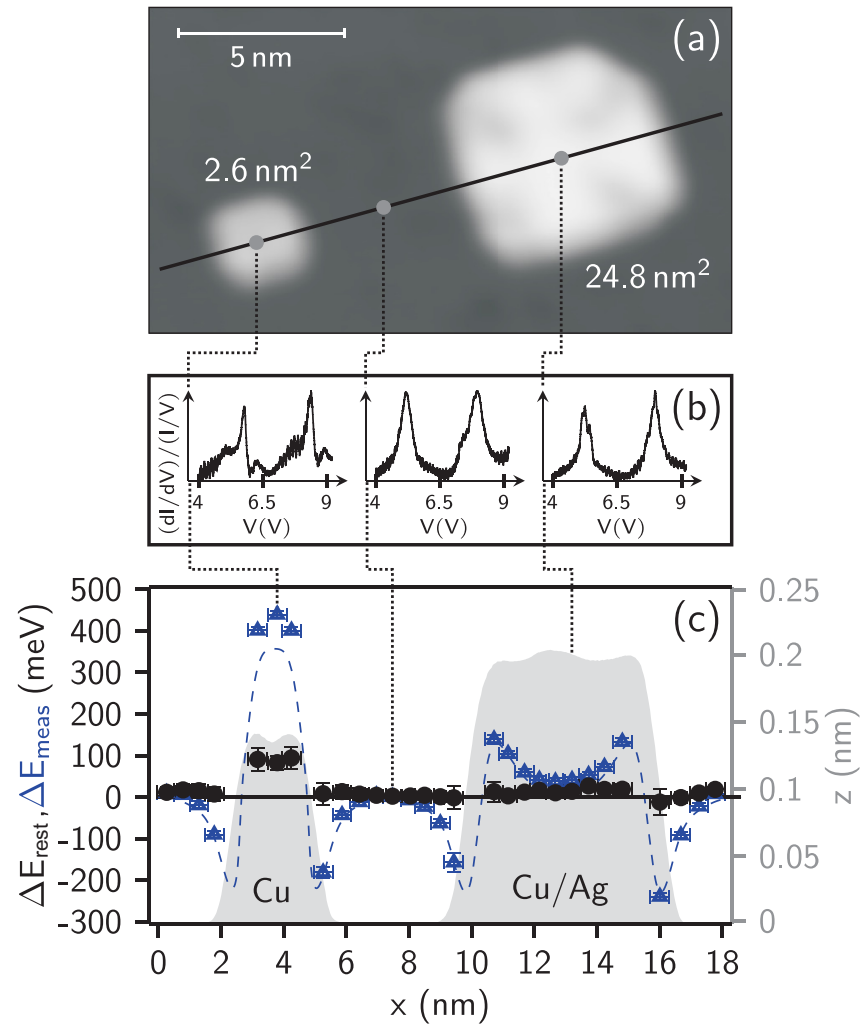

FIG. 4. (Color online) Differential conductivity $d I / d V$ spectra on two islands of different size: (a) Topography image of a small $\left(2.6 \mathrm{~nm}^{2}\right)$ and a large island $\left(24.8 \mathrm{~nm}^{2}\right)(I=44 \mathrm{pA}, V=0.2 \mathrm{~V})$. (b) Normalized $d I / d V$ spectra recorded at points marked in (a). (c) Energy shifts $\Delta E_{\text {meas }}$ of the 2nd image potential state with respect to the average value on the terrace (gray/blue triangles, left axis), and $\Delta E_{\text {rest }}$ corrected by the step effect (black circles, left axis); gray curve (right axis) depicts the island height vs position $x$ along the black line in (a). The dashed gray/blue line shows the energy correction function employed.

expected for quantum size confinement. Already a simple two-dimensional particle-in-a-box model predicts an energy shift that is inversely proportional to the island size and thus rationalizes the energy variations. However, this level of theory cannot explain the data quantitatively. Figure 3(b) illustrates the energy shift predicted by this model for squareshaped boxes with infinite potential wells. In order to obtain quantitative agreement, an unrealistically small effective mass $m^{*}$ would have to be anticipated. Also reasonable deviations from square-shaped islands do not improve the agreement with the experimental data. Similarly, it is unlikely that the assumption of an infinite potential well causes the deviation, because this assumption led to excellent agreement with a more accurate theory based on an elaborately parameterized model Hamiltonian for prototypical disk-shaped islands of monatomic height [16]. Thus quantum size confinement alone cannot explain the observed energy shift.

We now employ a correction scheme to obtain average energy shifts $\overline{\Delta E}_{\text {rest }}$ beyond quantum size confinement [Fig. 3(b), black circles]. The features at the island borders in Fig. 4(c) are indicative of the quantum size confinement, which result from scattering of the electrons at the step edges. 
A scattering at one of the edges is thereby independent from that at the other edges. In fact, similar shifts are measured at intrinsic surface steps that are well separated from other edges. The energy shifts close to such surface steps are thus utilized to separate that part of the island's energy shift that is due to quantum size confinement from the one due to atom density. To obtain a function that describes the confinement effect, a series of $d I / d V$ spectra are recorded perpendicular to intrinsic step edges of a bare $\mathrm{Ag}(100)$ surface and of bare $\mathrm{Cu}(111)$. The energy shift of each spectrum with respect to the terrace value is plotted vs the lateral distance from the step edge, and the energy shifts are fitted with cubic spline functions. A two-dimensional superposition of these functions for the four borders gives the energy shift due to the quantum size confinement [Fig. 1(c)]. These functions for the specific island size [see dashed line in Fig. 4(c)] are used to correct the measured values resulting in the remaining energy shifts $\Delta E_{\text {rest }}=\Delta E_{\text {meas }}-\Delta E_{\text {step }}$ [Fig. 4(c) and 3(b), black circles]. A similar procedure is applied to extract the relative line width $\bar{\Gamma}_{\text {rest }}$ [Fig. 3(a)].

For islands larger than $15 \mathrm{~nm}^{2}$ the energy is comparable to the terrace value within the experimental uncertainty. Such a small difference suggests a similar material and structure in the surface and the island. In fact, we proposed before that both the surface and islands larger than $7 \mathrm{~nm}^{2}$ consist of a $\mathrm{Cu} / \mathrm{Ag}$ alloy [17]. The small shift in energy is consistent with the vanishing shift of the work function for dilute alloys and the nonlinear dependence of the work function on alloy ratio for metal alloys [34]. The energy decreases slightly for island sizes ranging from 15 to $5 \mathrm{~nm}^{2}$. This energy decrease might reflect the dealloying of the islands leading eventually to the formation of pure copper islands at island sizes below $5 \mathrm{~nm}^{2}$ [34]. The exact processes during alloying and reasons for it demands a large scale calculation and further high resolution imaging during dealloying. Nonetheless, the earlier interpretation of dealloying [17] is corroborated here by an analysis of the linewidth, which is proportional to the reciprocal lifetime. It is abruptly reduced by a factor of 3 between 5 and $4 \mathrm{~nm}^{2}$ [Fig. 3(a)]. This corresponds to a tripling of the lifetime. Such a major change is indicative of a severe reduction of scattering centers.

Most interesting is the residual shift of the energy to up to $300 \mathrm{meV}$ for the pure copper islands that are smaller than 5 $\mathrm{nm}^{2}$ [shown in higher resolution in Fig. 3(c)]. According to our large-scale DFT calculations described previously [17,35], these nanoclusters are very different from an ideal (100) face: $\mathrm{Cu}$ islands containing 16 to 49 atoms turn out to be reconstructed with some of the $\mathrm{Cu}-\mathrm{Cu}$ distances significantly shortened [Fig. 3(c), insets]. As a consequence, the islands contain regions of (100) and (111) signature and the island atom density varies distinctly over this size range. This structural reordering is driven by the strain originating from the large $\mathrm{Cu} / \mathrm{Ag}$ lattice mismatch [35].
We define the island's density $\rho$ as the number of $\mathrm{Cu}$ atoms divided by the area of the bounding rectangle of the calculated sphere model for each reconstructed $\mathrm{Cu}$ island from our DFT calculations. Based on this definition $\rho \approx 15.3 \mathrm{~nm}^{-2}$ for $\mathrm{Cu}(100)$ and $\rho \approx 17.7 \mathrm{~nm}^{-2}$ for $\mathrm{Cu}(111)$. For the simulated $\mathrm{Cu}$ islands, the density increases with decreasing island size [Fig. 3(c), right axis]. In order to achieve a correlation between the experimentally obtained corrected energy shifts $\overline{\Delta E}_{\text {rest }}$ and the island atom density $\rho$, we now relate the density linearly to the 2PPE measured energy shifts of $(180 \pm 10) \mathrm{meV}$ [5,36] for $\mathrm{Cu}(100)$ and of $(460 \pm 10) \mathrm{meV}[5,37]$ for $\mathrm{Cu}(111)$, both of the 2nd image potential state and with respect to $\operatorname{Ag}(100)$ [38]. This procedure is justified by the fact that the work function and thus the image potential state energies were found to depend linearly on the surface atom density [39] and reach their undisturbed values in heteroepitaxial metal systems just after deposition of one metal monolayer [36]. Plotting the experimentally obtained energy shifts on this calibrated $\rho$ scale [Fig. 3(c), left axis] reveals an excellent agreement between the semiempirically predicted and the actually measured energy shift. The structural reordering with decreasing $\mathrm{Cu}$ island size is thus directly reflected in the energetic position of the image potential state. Consequently, this experiment offers the intriguing possibility for the local determination of the atomic density of nanostructures that cannot be resolved atomically, e.g., facets of size-selected nanoclusters [40].

In summary, the adatom density of $\mathrm{Cu}$ islands is determined in a size range from 1 to $5 \mathrm{~nm}^{2}$ for the $\mathrm{Cu} / \mathrm{Ag}(100)$ system via scanning tunneling spectroscopy of Stark-shifted image potential states. The sensitivity of the locally measured image potential state to subtle structural changes is corroborated by a quantitative comparison of the experimental energy shift to a structural reordering identified by density-functional theory. The degree of island reconstruction is directly linked to an energy shift of the 2nd image potential state after a confinement correction and can thus be determined with high spatial resolution (currently $\approx 0.3 \mathrm{~nm}$ ). Complementary to averaging techniques, this paves a way towards a hitherto inaccessible structural classification at the nanoscale. Such an analysis of the image potential state has not yet been applied to individual nano-objects. In contrast to the proof-of-principle here, high-level calculations are not necessary in future application of our approach. The thereby determined adatom density can be used as an important input for theoretical calculations that in turn may reveal the internal structure of the nanoclusters.

We thank the Rechenzentrum Garching (RZG) of the Max Planck Society for generous provision of CPU time. C.Z. acknowledges financial support by the Studienstiftung des deutschen Volkes.
[1] K. Tanaka, Materials 3, 4518 (2010); K. Zhou and Y. Li, Angew. Chem. Int. Ed. 51, 602 (2012).

[2] P. D. Nellist, M. F. Chisholm, N. Dellby, O. L. Krivanek, M. F. Murfitt, Z. S. Szilagyi, A. R. Lupini, A. Borisevich,
W. H. Sides, Jr., and S. J. Pennycook, Science 305, 1741 (2004).

[3] S. Van Aert, K. J. Batenburg, M. D. Rossell, R. Erni, and G. Van Tendeloo, Nature (London) 470, 374 (2011). 
[4] C.-C. Chen, C. Zhu, E. R. White, C.-Y. Chiu, M. C. Scott, B. C. Regan, L. D. Marks, Y. Huang, and J. Miao, Nature (London) 496, 74 (2013).

[5] E. V. Chulkov, V. M. Silkin, and P. M. Echenique, Surf. Sci. 437, 330 (1999).

[6] E. V. Chulkov, A. G. Borisov, J. P. Gauyacq, D. Sanchez-Portal, V. M. Silkin, V. P. Zhukov, and P. M. Echenique, Chem. Rev. 106, 4160 (2006).

[7] F. J. Himpsel and T. Fauster, J. Vac. Sci. Technol. A 2, 815 (1984); N. V. Smith, Rep. Prog. Phys. 51, 1227 (1988).

[8] M. Weinelt, J. Phys.: Condens. Matter 14, R1099 (2002); P. M. Echenique, R. Berndt, E. V. Chulkov, Th. Fauster, A. Goldmann, and U. Höfer, Surf. Sci. Rep. 52, 219 (2004); M. Winter, E. V. Chulkov, and U. Höfer, Phys. Rev. Lett. 107, 236801 (2011).

[9] P. Wahl, M. A. Schneider, L. Diekhöner, R. Vogelgesang, and K. Kern, Phys. Rev. Lett. 91, 106802 (2003); J. I. Pascual, C. Corriol, G. Ceballos, I. Aldazabal, H.-P. Rust, K. Horn, J. M. Pitarke, P. M. Echenique, and A. Arnau, Phys. Rev. B 75, 165326 (2007); P. Ruffieux, K. Ait-Mansour, A. Bendounan, R. Fasel, L. Patthey, P. Gröning, and O. Gröning, Phys. Rev. Lett. 102, 086807 (2009).

[10] C. L. Lin, S. M. Lu, W. B. Su, H. T. Shih, B. F. Wu, Y. D. Yao, C. S. Chang, and T. T. Tsong, Phys. Rev. Lett. 99, 216103 (2007); note that the state of "order 1" in this article corresponds to the 2nd image potential state.

[11] R. S. Becker, J. A. Golovchenko, and B. S. Swartzentruber, Phys. Rev. Lett. 55, 987 (1985).

[12] G. Binnig, K. H. Frank, H. Fuchs, N. Garcia, B. Reihl, H. Rohrer, F. Salvan, and A. R. Williams, Phys. Rev. Lett. 55, 991 (1985).

[13] F. Mohn, L. Gross, N. Moll, and G. Meyer, Nat. Nanotechnol. 7, 227 (2012).

[14] K. Schouteden and C. Van Haesendonck, Phys. Rev. Lett. 103, 266805 (2009).

[15] K. Schouteden and C. Van Haesendonck, Phys. Rev. Lett. 108, 076806 (2012).

[16] J. P. Gauyacq and A. G. Borisov, Surf. Sci. 600, 825 (2006); T. Hakala, M. J. Puska, A. G. Borisov, V. M. Silkin, N. Zabala, and E. V. Chulkov, Phys. Rev. B 75, 165419 (2007).

[17] C. Zaum, M. Rieger, K. Reuter, and K. Morgenstern, Phys. Rev. Lett. 107, 046101 (2011).

[18] M. Mehlhorn, H. Gawronski, L. Nedelmann, A. Grujic, and K. Morgenstern, Rev. Sci. Instrum. 78, 033905 (2007).

[19] K. Morgenstern, Phys. Status Solidi B 242, 773 (2005).

[20] J. Li, W.-D. Schneider, R. Berndt, and S. Crampin, Phys. Rev. Lett. 80, 3332 (1998).

[21] J. Henzl, K. Boom, and K. Morgenstern, J. Am. Chem. Soc. 135, 11501 (2013).

[22] S. Heidorn, C. Bertram, J. Koch, K. Boom, F. Matthaei, A. Safiei, J. Henzl, and K. Morgenstern, J. Phys. Chem. C 117, 16095 (2013).

[23] D. M. Eigler, C. P. Lutz, and W. E. Rudge, Nature (London) 352, 600 (1991).
[24] J. Repp, G. Meyer, S. M. Stojkovic, A. Gourdon, and C. Joachim, Phys. Rev. Lett. 94, 026803 (2005).

[25] While the STS operation often employs a lock-in technique to obtain a better signal-to-noise ratio with the disadvantage of modulation broadening, this is not necessary here. The excellent signal-to-noise ratio allows us to calculate $d z / d V$ or $d I / d V$ signals numerically from $z(V)$ or $I(V)$ measurements.

[26] M. Ziegler, N. Neel, A. Sperl, J. Kröger, and R. Berndt, Phys. Rev. B 80, 125402 (2009).

[27] J. A. Stroscio, R. M. Feenstra, and A. P. Fein, Phys. Rev. Lett. 57, 2579 (1986).

[28] D. B. Dougherty, P. Maksymovych, J. Lee, M. Feng, H. Petek, and J. T. Yates, Jr., Phys. Rev. B 76, 125428 (2007).

[29] A. Hanuschkin, D. Wortmann, and S. Blügel, Phys. Rev. B 76, 165417 (2007).

[30] S. Crampin, Phys. Rev. Lett. 95, 046801 (2005).

[31] S. Schintke, S. Messerli, M. Pivetta, F. Patthey, L. Libioulle, M. Stengel, A. De Vita, and W.-D. Schneider, Phys. Rev. Lett. 87, 276801 (2001)

[32] S. Joshi, D. Ecija, R. Koitz, M. Iannuzzi, A. P. Seitsonen, J. Hutter, H. Sachdev, S. Vijayaraghavan, F. Bischoff, J. V. Barth, and W. Auwrter, Nano Lett. 12, 5821 (2012).

[33] Note that the small variation in intensity on the large island in Fig. 1(b) is the standing waves of the laterally confined image potential state, which does not affect the energy position of the image potential state.

[34] Because of the nonlinear dependence of the work function on the alloy ratio [J. A. Rothschild and M. Eizenberg, Phys. Rev. B 81, 224201 (2010)] (and thus also of the measured image potential state energies), a quantitative modeling of the observed state energy decrease is not feasible with current theoretical descriptions.

[35] M. Rieger, Ph.D. thesis, Freie Universität Berlin, 2010.

[36] T. Fauster and W. Steinmann, in Electromagnetic Waves: Recent Developments in Research, edited by P. Halevi (Elsevier, Amsterdam, 1995), Vol. 2.

[37] A. Hotzel, G. Moos, K. Ishioka, M. Wolf, and G. Ertl, Appl. Phys. B 68, 615 (1999).

[38] Unfortunately, a direct DFT calculation of the image potential state is not feasible with present-day DFT exchange-correlation functionals [5,29], while more advanced $a b$ initio theory "beyond DFT" [6] is not yet computationally tractable for corresponding system sizes. The same holds for state-of-theart model Hamiltonian approaches [14], which are not yet applicable to the aforementioned complex island structures. In this situation, we instead aim to correlate the experimentally corrected energy shifts semiempirically with the most salient feature of the DFT island structures, namely, the distinctly varying island atom density.

[39] R. Smoluchowski, Phys. Rev. 60, 661 (1941); N. D. Lang and W. Kohn, Phys. Rev. B 3, 1215 (1971); A. W. Dweydari and C. H. B. Mee, Phys. Status Solidi A 27, 223 (1975).

[40] S. Messerli, S. Schintke, K. Morgenstern, A. Sanchez, U. Heiz, and W.-D. Schneider, Surf. Sci. 465, 331 (2000). 